\title{
Almost Sequence Spaces Derived by the Domain of the Matrix $A^{r}$
}

\author{
Ali Karaisa and Ümıt Karabıyık \\ Department of Mathematics-Computer Science, Faculty of Sciences, Necmettin Erbakan University, Meram Yerleşkesi, Meram, \\ 42090 Konya, Turkey
}

Correspondence should be addressed to Ali Karaisa; alikaraisa@hotmail.com

Received 9 May 2013; Revised 26 August 2013; Accepted 26 September 2013

Academic Editor: Feyzi Başar

Copyright (c) 2013 A. Karaisa and Ü. Karabiyık. This is an open access article distributed under the Creative Commons Attribution License, which permits unrestricted use, distribution, and reproduction in any medium, provided the original work is properly cited.

By using $A^{r}$, we introduce the sequence spaces $a_{f}^{r}, a_{f_{0}}^{r}$, and $a_{f_{s}}^{r}$ of normed space and $B K$-space and prove that $a_{f}^{r}$, $a_{f_{0}}^{r}$, and $a_{f s}^{r}$ are linearly isomorphic to the sequence spaces $f, f_{0}$, and $f_{s}$, respectively. Further, we give some inclusion relations concerning the spaces $a_{f}^{r}, a_{f_{0}}^{r}$, and the nonexistence of Schauder basis of the spaces $f s$ and $a_{f s}^{r}$ is shown. Finally, we determine the $\beta$ - and $\gamma$-duals of the spaces $a_{f}^{r}$ and $a_{f s}^{r}$. Furthermore, the characterization of certain matrix classes on new almost convergent sequence and series spaces has exhaustively been examined.

\section{Preliminaries, Background and Notation}

By $w$, we will denote the space of all real or complex valued sequences. Any vector subspace of $w$ is called sequence space. We will write $\ell_{\infty}, c_{0}, c$, and $\ell_{p}$ for the spaces of all bounded, null, convergent, and absolutely $p$-summable sequences, respectively, which are $B K$-space with the usual sup-norm defined by $\|x\|_{\infty}=\sup _{k}\left|x_{k}\right|$ and $\|x\|_{\ell_{p}}=\left(\sum_{k}\left|x_{k}\right|^{p}\right)^{1 / p}$, for $1<$ $p<\infty$, where, here and in what follows, the summation without limits runs from 0 to $\infty$. Further, we will write $b s$, cs for the spaces of all sequences associated with bounded and convergent series, respectively, which are $B K$-spaces with their natural norm [1].

Let $\mu$ and $\gamma$ be two sequence spaces and $A=\left(a_{n k}\right)$ an infinite matrix of real or complex numbers $a_{n k}$, where $n, k \in$ $\mathbb{N}$. Then, we say that $A$ defines a matrix mapping from $\mu$ into $\gamma$ and we denote it by writing that $A: \mu \rightarrow \gamma$ and if for every sequence $x=\left(x_{k}\right) \in \mu$ the sequence $A x=(A x)_{n}$, the $A$-transform of $x$ is in $\gamma$, where

$$
(A x)_{n}=\sum_{k} a_{n k} x_{k}, \quad(n \in \mathbb{N}) .
$$

The notation $(\mu: \gamma)$ denotes the class of all matrices $A$ such that $A: \mu \rightarrow \gamma$. Thus, $A \in(\mu: \gamma)$ if and only if the series on the right hand side of (1) converges for each $n \in \mathbb{N}$ and every $x \in \mu$ and we have $A x=\left\{(A x)_{n}\right\}_{n \in \mathbb{N}} \in \gamma$ for all $x \in \mu$.
The matrix domain $\mu_{A}$ of an infinite matrix $A$ in a sequence space $\mu$ is defined by

$$
\mu_{A}=\left\{x=\left(x_{k}\right) \in \omega: A x \in \mu\right\} .
$$

The approach constructing a new sequence space by means of the matrix domain of a particular triangle has recently been employed by several authors in many research papers. For example, they introduced the sequence spaces $(c)_{C_{1}}=\widetilde{c}$ in [2], $\left(\ell_{p}\right)_{A^{r}}=a_{p}^{r}$ and $\left(\ell_{\infty}\right)_{A^{r}}=a_{\infty}^{r}$ in [3], $\mu_{G}=$ $Z(u, v ; \mu)$ in [4], $\left(c_{0}\right)_{\Lambda}=c_{0}^{\lambda}$ and $c_{\Lambda}=c^{\lambda}$ in [5], and $\left(\ell_{p}\right)_{E^{r}}=e_{p}^{r}$ and $\left(\ell_{\infty}\right)_{E^{r}}=e_{\infty}^{r}$ in [6]. Recently, matrix domains of the generalized difference matrix $B(r, s)$ and triple band matrix $B(r, s, t)$ in the sets of almost null and almost convergent sequences have been investigated by Başar and Kirișçi [7] and Sönmez [8], respectively. Later, Kayaduman and Şengönül introduced some almost convergent spaces which are the matrix domains of the Riesz matrix and Cesàro matrix of order 1 in the sets of almost null and almost convergent sequences (see $[9,10])$.

We now focus on the sets of almost convergent sequences. A continuous linear functional $\phi$ on $\ell_{\infty}$ is called a Banach limit if (i) $\phi(x) \geqslant 0$ for $x=\left(x_{k}\right)$ and $x_{k} \geqslant 0$ for every $k$, (ii) $\phi\left(x_{\sigma(k)}\right)=\phi\left(x_{k}\right)$, where $\sigma$ is shift operator which is defined on $\omega$ by $\sigma(k)=k+1$, and (iii) $\phi(e)=1$, where $e=(1,1,1, \ldots)$. A sequence $x=\left(x_{k}\right) \in \ell_{\infty}$ is said to be almost convergent to 
the generalized limit $\alpha$ if all Banach limits of $x$ are $\alpha$ [11] and denoted by $f-\lim x=\alpha$. In other words, $f-\lim x_{k}=\alpha$ uniformly in $n$ if and only if

$$
\lim _{m \rightarrow \infty} \frac{1}{m+1} \sum_{k=0}^{m} x_{k+n} \quad \text { uniformly in } n .
$$

The characterization given above was proved by Lorentz in [11]. We denote the sets of all almost convergent sequences $f$ and series $f s$ by

$$
f=\left\{x=\left(x_{k}\right) \in \omega: \lim _{m \rightarrow \infty} t_{m n}(x)=\alpha \quad \text { uniformly in } n\right\},
$$

where

$$
\begin{gathered}
t_{m n}(x)=\sum_{k=0}^{m} \frac{1}{m+1} x_{k+n}, \quad t_{-1, n}=0, \\
f_{S}=\left\{x=\left(x_{k}\right) \in \omega:\right. \\
\exists l \in \mathbb{C} \ni \lim _{m \rightarrow \infty} \sum_{k=0}^{m} \sum_{j=0}^{n+k} \frac{x_{j}}{m+1}=l \\
\text { uniformly in } n\} .
\end{gathered}
$$

We know that the inclusions $c \subset f \subset \ell_{\infty}$ strictly hold. Because of these inclusions, norms $\|\cdot\|_{f}$ and $\|\cdot\|_{\infty}$ of the spaces $f$ and $\ell_{\infty}$ are equivalent. So the sets $f$ and $f_{0}$ are BK-spaces with the norm $\|x\|_{f}=\sup _{m, n}\left|t_{m n}(x)\right|$.

The rest of this paper is organized, as follows. We give foreknowledge on the main argument of this study and notations in this section. In Section 2, we introduce the almost convergent sequence and series spaces $a_{f_{s}}^{r}$ and $a_{f}^{r}$ which are the matrix domains of the $A^{r}$ matrix in the almost convergent sequence and series spaces $f s$ and $f$, respectively. In addition, we give some inclusion relations concerning the spaces $a_{f}^{r}$, $a_{f_{0}}^{r}$, and the non-existence of Schauder basis of the spaces $f s$ and $a_{f s}^{r}$ is shown to give certain theorems related to behavior of some sequences. In Section 3, we determine the beta- and gamma-duals of the spaces $a_{f}^{r}$ and $a_{f s}^{r}$ and characterize the classes $\left(\gamma: a_{f}^{r}\right),\left(a_{f}^{r}: \mu\right),\left(\delta: a_{f s}^{r}\right)$ and $\left(a_{f}^{r}: \theta\right)$, where $\gamma \in$ $\left\{c(p), c_{0}(p), \ell_{\infty}(p), c s, b s, f s, f, c, \ell_{\infty}\right\}, \mu \in\left\{c s, c, \ell_{\infty}\right\}, \delta \in$ $\{c s, f s, b s\}$, and $\theta \in\left\{f, c, f s, \ell_{\infty}\right\}$, where $c(p), c_{0}(p)$, and $\ell_{\infty}(p)$ denote the space of Maddox convergent, null and bounded sequence spaces defined by Maddox [12].

Lemma 1 (see [13]). The set $f$ s has no Schauder basis.

\section{The Sequence Spaces $a_{f}^{r}, a_{f_{0}}^{r}$, and $a_{f_{s}}^{r}$ Derived by the Domain of the Matrix $A^{r}$}

In the present section, we introduce the sequence spaces $a_{f}^{r}$, $a_{f_{0}}^{r}$, and $a_{f_{s}}^{r}$ as the set of all sequences such that $A^{r}$-transforms of them are in the spaces $f, f_{0}$, and $f s$, respectively. Further, this section is devoted to examination of the basic topological properties of the sets $a_{f}^{r}, a_{f_{0}}^{r}$, and $a_{f_{s}}^{r}$. Recently, Aydin and Başar [14] studied the sequence spaces $a_{c}^{r}$ and $a_{0}^{r}$ :

$$
\begin{aligned}
& a_{c}^{r}=\left\{x=\left(x_{k}\right) \in \omega: \lim _{n \rightarrow \infty} \frac{1}{n+1} \sum_{k=0}^{n}\left(1+r^{k}\right) x_{k} \text { exists }\right\}, \\
& a_{0}^{r}=\left\{x=\left(x_{k}\right) \in \omega: \lim _{n \rightarrow \infty} \frac{1}{n+1} \sum_{k=0}^{n}\left(1+r^{k}\right) x_{k}=0\right\},
\end{aligned}
$$

where $A^{r}$ denotes the matrix $A^{r}=\left(a_{n k}^{r}\right)$ defined by

$$
a_{n k}^{r}= \begin{cases}\frac{1+r^{k}}{n+1} & (0 \leqslant k \leqslant n), \\ 0 & (k>n) .\end{cases}
$$

Now we introduce the sequence spaces $a_{f}^{r}$, $a_{f}^{r}$, and $a_{f_{s}}^{r}$ as the sets of all sequences such that their $A^{r}$-transforms are in the spaces $f, f_{0}$, and $f s$, respectively; that is,

$$
\begin{aligned}
& a_{f}^{r}=\left\{x=\left(x_{k}\right) \in \omega:\right. \\
& \exists \alpha \in \mathbb{C} \ni \lim _{m \rightarrow \infty} \sum_{k=0}^{m} \frac{1}{m+1} \sum_{i=0}^{k} \frac{1}{k+1}\left(1+r^{i}\right) x_{n+i} \\
& =\alpha \text { uniformly in } n\} \text {, } \\
& a_{f_{0}}^{r}=\left\{x=\left(x_{k}\right) \in \omega:\right. \\
& \lim _{m \rightarrow \infty} \sum_{k=0}^{m} \frac{1}{m+1} \sum_{i=0}^{k} \frac{1+r^{i}}{k+1} x_{n+i}=0 \\
& \text { uniformly in } n\} \text {, } \\
& a_{f s}^{r}=\left\{x=\left(x_{k}\right) \in \omega:\right. \\
& \exists \beta \in \mathbb{C} \ni \lim _{m \rightarrow \infty} \sum_{k=0}^{m} \sum_{j=0}^{n+k} \sum_{i=0}^{j} \frac{1+r^{j}}{i+1} x_{n+j} \\
& =\beta \quad \text { uniformly in } n\} \text {. }
\end{aligned}
$$

We can redefine the spaces $a_{f_{s}}^{r}, a_{f}^{r}$, and $a_{f_{0}}^{r}$ by the notation of (2):

$$
a_{f_{0}}^{r}=\left(f_{0}\right)_{A^{r}}, \quad a_{f}^{r}=f_{A^{r}}, \quad a_{f_{s}}^{r}=\left(f_{s}\right)_{A^{r}} .
$$

It is known by Başar [15] that the method is regular for $0<$ $r<1$. We assume unless stated otherwise that $0<r<1$. 
Define the sequence $y=\left(y_{k}\right)$, which will be frequently used, as the $A^{r}$-transform of a sequence $x=\left(x_{k}\right)$; that is,

$$
y_{k}(r)=\sum_{i=0}^{k} \frac{1+r^{i}}{k+1} x_{i}(k \in \mathbb{N}) \text {. }
$$

Theorem 2. The spaces $a_{f}^{r}$ and $a_{f s}^{r}$ have no Schauder basis.

Proof. Since it is known that the matrix domain $\mu_{A}$ of a normed sequence space $\mu$ has a basis if and only if $\mu$ has a basis whenever $A=\left(a_{n k}\right)$ is a triangle [16, Remark 2.4] and the space $f$ has no Schauder basis by [7, Corollary 3.3], we have that $a_{f}^{r}$ has no Schauder basis. Since the set $f s$ has no basis in Lemma 1, $a_{f s}^{r}$ has no Schauder basis.

\section{Theorem 3. The following statements hold.}

(i) The sets $a_{f}^{r}$ and $a_{f_{0}}^{r}$ are linear spaces with the coordinatewise addition and scalar multiplication which are $B K$-spaces with the norm

$$
\|x\|_{a_{f}^{r}}=\sup _{m}\left|\sum_{k=0}^{m} \frac{1}{m+1} \sum_{i=0}^{k} \frac{1+r^{i}}{k+1} x_{i+n}\right| .
$$

(ii) The set $a_{f s}^{r}$ is a linear space with the coordinatewise addition and scalar multiplication which is a BK-space with the norm

$$
\|x\|_{a_{f s}^{r}}=\sup _{m}\left|\sum_{k=0}^{m} \frac{1}{m+1} \sum_{j=0}^{k+n} \sum_{i=0}^{j} \frac{1+r^{i}}{j+1} x_{i}\right| .
$$

Proof. Since the second part can be similarly proved, we only focus on the first part. Since the sequence spaces $f$ and $f_{0}$ endowed with the norm $\|\cdot\|_{\infty}$ are $B K$-spaces (see [1, Example 7.3.2(b)] $)$ and the matrix $A^{r}=\left(a_{n k}^{r}\right)$ is normal, Theorem 4.3.2 of Wilansky [17, p.61] gives the fact that the spaces $a_{f}^{r}$ and $a_{f_{0}}^{r}$ are $B K$-spaces with the norm in (11).

Now, we may give the following theorem concerning the isomorphism between our spaces and the sets $f, f_{0}$, and $f s$.

Theorem 4. The sequence spaces $a_{f}^{r}, a_{f_{0}}^{r}$, and $a_{f s}^{r}$ are linearly isomorphic to the sequence spaces $f, f_{0}$, and $f s$, respectively; that is, $a_{f}^{r} \cong f, a_{f_{0}}^{r} \cong f_{0}$, and $a_{f_{s}}^{r} \cong f s$.

Proof. To prove the fact that $a_{f}^{r} \cong f$, we should show the existence of a linear bijection between the spaces $a_{f}^{r}$ and $f$. Consider the transformation $T$ defined with the notation of (2) from $a_{f}^{r}$ to $f$ by $x \mapsto y=T x=A^{r} x$. The linearity of $T$ is clear. Further, it is clear that $x=\theta$ whenever $T x=\theta$, and hence, $T$ is injective.

Let $y=\left(y_{k}\right) \in a_{f}^{r}$, and define the sequence $x=\left(x_{k}(r)\right)$ by

$$
x_{k}=\frac{1}{1+r^{k}}\left[(k+1) y_{k}-k y_{k-1}\right] \quad \text { for each } k \in \mathbb{N} \text {, }
$$

whence

$$
\begin{aligned}
f_{A^{r}}- & \lim x \\
= & \lim _{m \rightarrow \infty} \sum_{k=0}^{m} \frac{1}{m+1} \\
& \times \sum_{i=0}^{k} \frac{\left(1+r^{i}\right) x_{i+n}}{1+k} \text { uniformly in } n \\
= & \lim _{m \rightarrow \infty} \sum_{k=0}^{m} \frac{1}{m+1} \\
& \times \sum_{i=0}^{k} \frac{\left(1+r^{i}\right)\left[1 /\left(1+r^{i}\right)\left(y_{i+n}(k+1)-y_{i+n-1} k\right)\right]}{1+k} \quad \text { uniformly in } n \\
= & \lim _{m \rightarrow \infty} \frac{1}{m+1} \sum_{k=0}^{m} y_{k+n} \quad \text { uniformly in } n \\
= & f-\lim y
\end{aligned}
$$

which implies that $x \in a_{f}^{r}$. As a result, $T$ is surjective. Hence, $T$ is a linear bijection which implies that the spaces $a_{f}^{r}$ and $f$ are linearly isomorphic, as desired. Similarly, the isomorphisms $a_{f_{0}}^{r} \cong f_{0}$ and $a_{f_{s}}^{r} \cong f s$ can be proved.

Theorem 5. The inclusion $f \subset a_{f}^{r}$ strictly holds.

Proof. Let $x=\left(x_{k}\right) \in c$. Since $c \subset f, x \in f$. Because $A^{r}$ is regular for $0<r<1, A^{r} x \in c$. Therefore, since $\lim A^{r} x=f-$ $\lim A^{r} x$, we see that $x \in a_{f}^{r}$. So we have that the inclusion $f \subset$ $a_{f}^{r}$ holds. Further, consider the sequence $t=\left(t_{k}(r)\right)$ defined by $t_{k}(r)=(2 k+1) /\left(1+r^{k}\right)(-1)^{k} \forall k \in \mathbb{N}$. Then, since $A^{r} \mathrm{t}=$ $(-1)^{n} \in f, x \in a_{f}^{r}$. One can easily see that $t \notin f$. Thus, $t \in a_{f}^{r} \backslash$ $f$, and this completes the proof.

Theorem 6. The sequence spaces $a_{f}^{r}$ and $\ell_{\infty}$ overlap, but neither of them contains the other.

Proof. Let us consider the sequence $u=\left(u_{k}(r)\right)$ defined by $u_{k}(r)=1 /\left(1+r^{k}\right)$ for all $\mathbb{N}$. Then, since $A^{r} u=e \in f, u \in a_{f}^{r}$. It is clear that $u \in \ell_{\infty}$. This means that the sequence spaces $a_{f}^{r}$ and $\ell_{\infty}$ are not disjoint. Now, we show that the sequence space $a_{f}^{r}$ and $\ell_{\infty}$ do not include each other. Let us consider the sequence $t=\left(t_{k}(r)\right)$ defined as in proof of Theorem 5 above and $z=\left(z_{k}(r)\right)=\left(0, \ldots, 0,1 /\left(1+r^{101}\right), \ldots, 1 /\left(1+r^{110}\right)\right.$, $\left.0, \ldots, 0,1 /\left(1+r^{211}\right), \ldots, 1 /\left(1+r^{231}\right), 0, \ldots, 0, \ldots\right)$ where the blocks of 0 's are increasing by factors of 100 and the blocks of $1 /\left(1+r^{k}\right)$ 's are increasing by factors of 10 . Then, since $A^{r} t=$ $(-1)^{n} \in f, t \in a_{f}^{r}$, but $t \notin \ell_{\infty}$. Therefore, $t \in a_{f}^{r} \backslash \ell_{\infty}$. Also, the sequence $z \notin a_{f}^{r}$ since $A^{r} z=(0, \ldots, 0,1, \ldots, 1,0, \ldots, 0,1, \ldots$, $1,0, \ldots, 0, \ldots) \notin f$ where the blocks of 0 's are increasing by factors of 100 and the blocks of 1's are increasing by factors of 10 , but $z$ is bounded. This means that $z \in \ell_{\infty} \backslash a_{f}^{r}$. Hence, the 
sequence spaces $a_{f}^{r}$ and $\ell_{\infty}$ overlap, but neither of them contains the other. This completes the proof.

Theorem 7. Let the spaces $a_{f_{0}}^{r}, a_{c}^{r}$, and $a_{f}^{r}$ be given. Then,

(i) $a_{f_{0}}^{r} \subset a_{f}^{r}$ strictly hold;

(ii) $a_{c}^{r} \subset a_{f}^{r}$ strictly hold.

Proof. (i) Let $x=\left(x_{k}\right) \in a_{f_{0}}^{r}$ which means that $A^{r} x \in f_{0}$. Since $f_{0} \subset f, A^{r} x \in f$. This implies that $x \in a_{f}^{r}$. Thus, we have $a_{f_{0}}^{r} \subset a_{f}^{r}$.

Now, we show that this inclusion is strict. Let us consider the sequence $u=\left(u_{k}(r)\right)$ defined as in proof of Theorem 6 for all $k \in \mathbb{N}$. Consider the following:

$$
\begin{aligned}
f_{A^{r}}-\lim u & =\lim _{m \rightarrow \infty} \sum_{k=0}^{m} \frac{1}{m+1} \sum_{i=0}^{k} \frac{1+r^{i}}{k+1} u_{i+n} \\
& =\lim _{m \rightarrow \infty} \sum_{k=0}^{m} \frac{1}{m+1}=e=(1,1, \ldots)
\end{aligned}
$$

which means that $u \in a_{f}^{r} \backslash a_{f_{0}}^{r}$; that is to say, the inclusion is strict.

(ii) Let $x=\left(x_{k}\right) \in a_{c}^{r}$ which means that $A^{r} x \in c$. Since $c \subset$ $f, A^{r} x \in f$. This implies that $x \in a_{f}^{r}$. Thus, we have $a_{c}^{r} \subset a_{f}^{r}$. Furthermore, let us consider the sequence $t=\left\{t_{k}(r)\right\}$ defined as in proof of Theorem 5 for all $k \in \mathbb{N}$. Then, since $A^{r} t=(-1)^{n} \in f \backslash c, t \in a_{f}^{r} \backslash a_{c}^{r}$. This completes the proof.

\section{Certain Matrix Mappings on the Sets $a_{f}^{r}, a_{f_{s}}^{r}$ and Some Duals}

In this section, we will characterize some matrix transformations between the spaces of $A^{r}$ almost convergent sequence and almost convergent series in addition to paranormed and classical sequence spaces after giving $\beta$ - and $\gamma$-duals of the spaces $a_{f s}^{r}$ and $a_{f}^{r}$. We start with the definition of the beta- and gamma-duals.

If $x$ and $y$ are sequences and $X$ and $Y$ are subsets of $\omega$, then we write $x \cdot y=\left(x_{k} y_{k}\right)_{k=0}^{\infty}, x^{-1} * Y=\{a \in \omega: a \cdot x \in Y\}$ and

$$
M(X, Y)=\bigcap_{x \in X} x^{-1} * Y=\{a: a \cdot x \in Y \forall x \in X\}
$$

for the multiplier space of $X$ and $Y$. One can easily observe for a sequence space $Z$ with $Y \subset Z$ and $Z \subset X$ that inclusions $M(X, Y) \subset M(X, Z)$ and $M(X, Y) \subset M(Z, Y)$ hold, respectively. The $\alpha$-, $\beta$-, and $\gamma$-duals of a sequence space, which are, respectively, denoted by $X^{\alpha}, X^{\beta}$, and $X^{\gamma}$, are defined by

$X^{\alpha}=M\left(X, \ell_{1}\right), \quad X^{\beta}=M(X, c s), \quad X^{\gamma}=M(X, b s)$.

It is obvious that $X^{\alpha} \subset X^{\beta} \subset X^{\gamma}$. Also, it can easily be seen that the inclusions $X^{\alpha} \subset Y^{\alpha}, X^{\beta} \subset Y^{\beta}$, and $X^{\gamma} \subset Y^{\gamma}$ hold whenever $Y \subset X$.
Lemma 8 (see [18]). $A=\left(a_{n k}\right) \in\left(f: \ell_{\infty}\right)$ if and only if

$$
\sup _{n} \sum_{k}\left|a_{n k}\right|<\infty \text {. }
$$

Lemma 9 (see [18]). $A=\left(a_{n k}\right) \in(f: c)$ if and only if (18) holds and there are $\alpha, \alpha_{k} \in \mathbb{C}$ such that

$$
\begin{gathered}
\lim _{n \rightarrow \infty} a_{n k}=\alpha_{k} \quad \forall k \in \mathbb{N}, \\
\lim _{n \rightarrow \infty} \sum_{k} a_{n k}=\alpha, \\
\lim _{n \rightarrow \infty} \sum_{k}\left|\Delta\left(a_{n k}-\alpha_{k}\right)\right|=0 .
\end{gathered}
$$

Theorem 10. Define the sets $t_{1}^{r}$ and $t_{2}^{r}$ by

$$
\begin{gathered}
t_{1}^{r}=\left\{a=\left(a_{k}\right) \in \omega: \sum_{k}\left|\Delta\left(\frac{a_{k}}{1+r^{k}}\right)(k+1)\right|<\infty\right\}, \\
t_{2}^{r}=\left\{a=\left(a_{k}\right) \in \omega: \sup _{k}\left|\frac{a_{k}(k+1)}{1+r^{k}}\right|<\infty\right\},
\end{gathered}
$$

where $\Delta\left(a_{k} /\left(1+r^{k}\right)\right)=a_{k} /\left(1+r^{k}\right)-a_{k+1} /\left(1+r^{k+1}\right)$ for all $k \epsilon$ $\mathbb{N}$. Then $\left(a_{f}^{r}\right)^{\gamma}=t_{1}^{r} \cap t_{2}^{r}$.

Proof. Take any sequence $a=\left(a_{k}\right) \in \omega$, and consider the following equality:

$$
\begin{aligned}
\sum_{k=0}^{n} a_{k} x_{k}= & \sum_{k=0}^{n} a_{k}\left[\sum_{i=k-1}^{k}(-1)^{k-j} \frac{i+1}{1+r^{i}} y_{i}\right] \\
= & \sum_{k=0}^{n-1} \Delta\left(\frac{a_{k}}{1+r^{k}}\right)(k+1) y_{k} \\
& +\frac{n+1}{1+r^{n}} a_{n} y_{n} \\
= & (T y)_{n}
\end{aligned}
$$

where $T=\left\{t_{n k}^{r}\right\}$ is

$$
t_{n k}^{r}= \begin{cases}\Delta\left(\frac{a_{k}}{1+r^{k}}\right)(k+1) & (0 \leqslant k \leqslant n-1) \\ \frac{n+1}{1+r^{n}} a_{n} & (k=n) \\ 0 & (k>n)\end{cases}
$$

for all $k, n \in \mathbb{N}$. Thus, we deduce from (23) that $a x=\left(a_{k} x_{k}\right) \in$ bs whenever $x=\left(x_{k}\right) \in a_{f}^{r}$ if and only if $T y \in \ell_{\infty}$ whenever $y=\left(y_{k}\right) \in f$ where $T=\left\{t_{n k}^{r}\right\}$ is defined in (24). Therefore, with the help of Lemma $8,\left(a_{f}^{r}\right)^{\gamma}=t_{1}^{r} \cap t_{2}^{r}$.

Theorem 11. The $\beta$-dual of the space $a_{f}^{r}$ is the intersection of the sets

$$
\begin{gathered}
t_{3}^{r}=\left\{a=\left(a_{k}\right) \in \omega: \lim _{n \rightarrow \infty} \sum_{k}\left|\Delta\left(t_{n k}^{r}-\alpha_{k}\right)\right|=0\right\}, \\
t_{4}^{r}=\left\{a=\left(a_{k}\right) \in \omega:\left(\frac{k+1}{1+r^{k}} a_{k}\right) \in c s\right\},
\end{gathered}
$$

where $\lim _{n \rightarrow \infty} t_{n k}^{r}=\alpha_{k}$ for all $k \in \mathbb{N}$. Then, $\left(a_{f}^{r}\right)^{\beta}=t_{3}^{r} \cap t_{4}^{r}$. 
Proof. Let us take any sequence $a \in \omega$. By (23), $a x=\left(a_{k} x_{k}\right) \in$ cs whenever $x=\left(x_{k}\right) \in a_{f}^{r}$ if and only if $T y \in c$ whenever $y=$ $\left(y_{k}\right) \in f$. It is obvious that the columns of that matrix $T$ in $c$ where $T=\left\{t_{n k}^{r}\right\}$ defined in (24), we derive the consequence by Lemma 9 that $\left(a_{f}^{r}\right)^{\beta}=t_{3}^{r} \cap t_{4}^{r}$.

Theorem 12. The $\gamma$-dual of the space $a_{f_{s}}^{r}$ is the intersection of the sets

$$
\begin{gathered}
c_{1}^{r}=\left\{a=\left(a_{k}\right) \in \omega: \sum_{k} \mid \Delta\left[\Delta\left(\frac{a_{k}}{1+r^{k}}\right)(k+1)\right.\right. \\
\left.\left.+\frac{a_{k}}{1+r^{k}}(k+1)\right] \mid<\infty\right\}, \\
c_{2}^{r}=\left\{a=\left(a_{k}\right) \in \omega:\left(\frac{a_{k}(k+1)}{1+r^{k}}\right) \in c_{0}\right\} .
\end{gathered}
$$

In other words, we have $\left(a_{f_{s}}^{r}\right)^{\gamma}=c_{1}^{r} \cap c_{2}^{r}$.

Proof. We obtain from (23) that $a x=\left(a_{k} x_{k}\right) \in b s$ whenever $x=\left(x_{k}\right) \in a_{f s}^{r}$ if and only if $T y \in \ell_{\infty}$ whenever $y=\left(y_{k}\right) \in f_{s}$, where $T=\left\{t_{n k}^{r}\right\}$ is defined in (24). Therefore, by Lemma 19 (viii), $\left(a_{f_{s}}^{r}\right)^{\gamma}=c_{1}^{r} \cap c_{2}^{r}$.

Theorem 13. Define the set $c_{3}^{r}$ by

$$
c_{3}^{r}=\left\{a=\left(a_{k}\right) \in \omega: \lim _{n \rightarrow \infty} \sum_{k}\left|\Delta^{2}\left(t_{n k}^{r}\right)\right| \text { exists }\right\} .
$$

Then, $\left(a_{f_{s}}^{r}\right)^{\beta}=c_{1}^{r} \cap c_{2}^{r} \cap c_{3}^{r}$.

Proof. This may be obtained in the same way as mentioned in the proof of Theorem 12 with Lemma 19(viii) instead of Lemma 19(vii). So we omit details.

For the sake of brevity, the following notations will be used:

$$
\begin{gathered}
a(n, k, m)=\frac{1}{m+1} \sum_{i=0}^{m} a_{n+i, k}, \quad a(n, k)=\sum_{i=0}^{n} a_{i k}, \\
\widehat{a}_{n k}=\Delta\left(\frac{a_{n k}}{1+r^{k}}\right)(k+1)=\left(\frac{a_{n k}}{1+r^{k}}-\frac{a_{n, k+1}}{1+r^{k+1}}\right)(k+1), \\
\Delta a_{n k}=a_{n, k}-a_{n, k+1}, \quad \widetilde{a}_{n k}=\sum_{j=0}^{n} \frac{\left(1+r^{j}\right) e_{j k}}{n+1},
\end{gathered}
$$

for all $k, n \in \mathbb{N}$. Assume that the infinite matrices $A=\left(a_{n k}\right)$ and $B=\left(b_{n k}\right)$ map the sequences $x=\left(x_{k}\right)$ and $y=\left(y_{k}\right)$ which are connected with relation (10) to the sequences $u=\left(u_{n}\right)$ and $v=\left(v_{n}\right)$, respectively; that is,

$$
\begin{aligned}
& u_{n}=(A x)_{n}=\sum_{k} a_{n k} x_{k} \quad \forall n \in \mathbb{N}, \\
& v_{n}=(B y)_{n}=\sum_{k} b_{n k} y_{k} \quad \forall n \in \mathbb{N} .
\end{aligned}
$$

One can easily conclude here that the method $A$ is directly applied to the terms of the sequence $x=\left(x_{k}\right)$, while the method $B$ is applied to the $A^{r}$-transform of the sequence $x=$ $\left(x_{k}\right)$. So the methods $A$ and $B$ are essentially different.

Now, suppose that the matrix product $B A^{r}$ exists which is a much weaker assumption than the conditions on the matrix $B$ belonging to any matrix class, in general. It is not difficult to see that the sequence in (30) reduces to the sequence in (29) under the application of formal summation by parts. This leads us to the fact that $B A^{r}$ exists and is equal to $A$ and $\left(B A^{r}\right) x=B\left(A^{r} x\right)$ formally holds if one side exists. This statement is equivalent to the following relation between the entries of the matrices $A=\left(a_{n k}\right)$ and $B=\left(b_{n k}\right)$ which are connected with the relation

$$
\begin{aligned}
& \widehat{a}_{n k}=b_{n k}=\Delta\left(\frac{a_{n k}}{1+r^{k}}\right)(k+1) \text { or } \\
& a_{n k}=\left(1+r^{k}\right) \sum_{j=k}^{\infty} \frac{b_{n j}}{1+j}
\end{aligned}
$$

$\forall k, n \in \mathbb{N}$.

Note that the methods $A$ and $B$ are not necessarily equivalent since the order of summation may not be reversed. We now give the following fundamental theorem connected with the matrix mappings on/into the almost convergent spaces $a_{f}^{r}$ and $a_{f s}^{r}$.

Theorem 14. Suppose that the entries of the infinite matrices $A=\left(a_{n k}\right)$ and $B=\left(b_{n k}\right)$ are connected with relation (31) for all $k, n \in \mathbb{N}$, and let $\lambda$ be any given sequence space. Then, $A \in\left(a_{f}^{r}\right.$ : $\lambda$ ) if and only if

$$
\begin{gathered}
B \in(f: \lambda), \\
\left\{\frac{n+1}{1+r^{k}} a_{n k}\right\}_{k \in \mathbb{N}} \in c_{0} .
\end{gathered}
$$

Proof. Suppose that $A=\left(a_{n k}\right)$ and $B=\left(b_{n k}\right)$ are connected with the relation (31), and let $\lambda$ be any given sequence space, and keep in mind that the spaces $a_{f}^{r}$ and $f$ are norm isomorphic.

Let $A \in\left(a_{f}^{r}: \lambda\right)$, and take any sequence $x \in a_{f}^{r}$, and keep in mind that $y=A^{r} x$. Then, $\left(a_{n k}\right)_{k \in \mathbb{N}} \in\left(a_{f}^{r}\right)^{\beta}$; that is, (32) holds for all $n \in \mathbb{N}$ and $B A^{r}$ exists which implies that $\left(b_{n k}\right)_{k \in \mathbb{N}} \in \ell_{1}=f^{\beta}$ for each $n \in \mathbb{N}$. Thus, By exists for all $y \in$ $f$, and thus, we have $m \rightarrow \infty$ in the equality

$$
\sum_{k=0}^{m} b_{n k} y_{k}=\sum_{k=0}^{m} \sum_{j=k}^{m}\left(1+r^{k}\right) \frac{b_{n j}}{1+j} x_{k}
$$

for all $m, n \in \mathbb{N}$, and we have (31) $B y=A x$ which means that $B \in(f: \lambda)$. On the other hand, assume that (32) holds and $B \in(f: \lambda)$. Then, we have $\left(b_{n k}\right)_{k \in \mathbb{N}} \in \ell_{1}$ for all $n \in \mathbb{N}$ which 
gives together with $\left(v_{n k}\right)_{k \in \mathbb{N}} \in\left(a_{f}^{r}\right)^{\beta}$ for each $n \in \mathbb{N}$ that $A x$ exists. Then, we obtain from the equality

$$
\begin{aligned}
\sum_{k=0}^{m} a_{n k} x_{k} & =\sum_{k=0}^{m-1} \Delta\left(\frac{a_{n k}}{1+r^{k}}\right)(k+1) y_{k}+\frac{m+1}{1+r^{m}} a_{n m} y_{m} \\
& =\sum_{k=0}^{m} b_{n k} y_{k}
\end{aligned}
$$

for all $m, n \in \mathbb{N}$, as $m \rightarrow \infty$, that $A x=B y$, and this shows that $A \in\left(a_{f}^{r}: \lambda\right)$.

Theorem 15. Suppose that the entries of the infinite matrices $E=\left(e_{n k}\right)$ and $F=\left(f_{n k}\right)$ are connected with the relation

$$
f_{n k}=\tilde{a}_{n k}
$$

for all $m, n \in \mathbb{N}$ and $\lambda$ is any given sequence space. Then, $E \in$ $\left(\lambda: a_{f}^{r}\right)$ if and only if $F \in(\lambda: f)$.

Proof. Let $x=\left(x_{k}\right) \in \lambda$, and consider the following equality:

$$
\sum_{j=0}^{n} \sum_{k=0}^{m} \frac{1+r^{j}}{n+1} e_{j k} x_{k}=\sum_{k=0}^{m} f_{n k} x_{k}
$$

for all $k, m, n \in \mathbb{N}$, which yields as $m \rightarrow \infty$ that $E x \in a_{f}^{r}$ whenever $x \in \lambda$ if and only if $F x \in f$ whenever $x \in \lambda$. This step completes the proof.

Theorem 16. Let $\lambda$ be any given sequence space, and the matrices $A=\left(a_{n k}\right)$ and $B=\left(b_{n k}\right)$ are connected with the relation (31). Then, $A \in\left(a_{f_{s}}^{r}: \lambda\right)$ if and only if $B \in\left(f_{s}: \lambda\right)$ and $\left(a_{n k}\right)_{k \in \mathbb{N}} \in$ $\left(a_{f s}^{r}\right)^{\beta}$ for all $n \in \mathbb{N}$.

Proof. The proof is based on the proof of Theorem 14 .

Theorem 17. Let $\lambda$ be any given sequence space, and the elements of the infinite matrices $E=\left(e_{n k}\right)$ and $F=\left(f_{n k}\right)$ are connected with relation (35). Then, $E=\left(e_{n k}\right) \in\left(\lambda: a_{f_{s}}^{r}\right)$ if and only if $F \in(\lambda: f s)$.

Proof. The proof is based on the proof of Theorem 15.

By Theorems 14, 15, 16, and 17, we have quite a few outcomes depending on the choice of the space $\lambda$ to characterize certain matrix mappings. Hence, by the help of these theorems, the necessary and sufficient conditions for the classes $\left(a_{f}^{r}: \lambda\right),\left(\lambda: a_{f}^{r}\right),\left(a_{f s}^{r}: \lambda\right)$ and $\left(\lambda: a_{f_{s}}^{r}\right)$ may be derived by replacing the entries of $A$ and $B$ by those of $B=A\left(A^{r}\right)^{-1}$, and $F=A^{r} E$, respectively, where the necessary and sufficient conditions on the matrices $E$ and $F$ are read from the concerning results in the existing literature

Lemma 18. Let $A=\left(a_{n k}\right)$ be an infinite matrix. Then, the following statements hold: (i) $A \in\left(c_{0}(p): f\right)$ if and only if

$$
\exists N>1 \ni \sup _{m \in \mathbb{N}} \sum_{k}|a(n, k, m)| N^{1 / p_{k}}<\infty, \quad \forall n \in \mathbb{N},
$$

$\exists \alpha_{k} \in \mathbb{C} \quad \forall k \in \mathbb{N} \ni \lim _{m \rightarrow \infty} a(n, k, m)=\alpha_{k} \quad$ uniformly in $n ;$

(ii) $A \in(c(p): f)$ if and only if (37) and

$\exists \alpha \in \mathbb{C} \ni \lim _{m \rightarrow \infty} \sum_{k} a(n, k, m)=\alpha \quad$ uniformly in $n$

(iii) $A \in\left(\ell_{\infty}(p): f\right)$ if and only if (37) and

$$
\exists N>1 \ni \lim _{m \rightarrow \infty} \sum_{k}\left|a(n, k, m)-\alpha_{k}\right| N^{1 / p_{k}}=0
$$

uniformly in $n$.

Lemma 19. Let $A=\left(a_{n k}\right)$ be an infinite matrix. Then, the following statements hold:

(i) (Duran, [19]) $A \in\left(\ell_{\infty}: f\right)$ if and only if (18) holds and

$$
f-\lim a_{n k}=\alpha_{k} \text { exists for each fixed } k \text {, }
$$

$$
\lim _{m \rightarrow \infty} \sum_{k}\left|a(n, k, m)-\alpha_{k}\right|=0 \quad \text { uniformly in } n \text {; }
$$

(ii) (King, [20]) $A \in(c: f)$ if and only if (18), (40) hold and

$$
f-\lim \sum_{k} a_{n k}=\alpha
$$

(iii) (Başar and Çolak, [21]) $A \in(c s: f)$ if and only if (40) holds and

$$
\sup _{n \in \mathbb{N}} \sum_{k}\left|\Delta a_{n k}\right|<\infty
$$

(iv) (Başar and Çolak, [21]) $A \in(b s: f)$ if and only if (40), (43) hold and

$$
\lim _{k} a_{n k}=0 \text { exists for each fixed } n,
$$

$\lim _{q \rightarrow \infty} \sum_{k} \frac{1}{q+1} \sum_{i=0}^{q}\left|\Delta\left[a(n+i, k)-\alpha_{k}\right]\right|=0 \quad$ uniformly in $n$;

(v) (Duran, [19]) $A \in(f: f)$ if and only if (18), (40), and (42) hold and

$\lim _{m \rightarrow \infty} \sum_{k}\left|\Delta\left[a(n, k, m)-\alpha_{k}\right]\right|=0 \quad$ uniformly in $n$

(vi) (Başar, [22]) $A \in(f s: f)$ if and only if (40), (44), (46), and (45) hold; 
(vii) (Öztürk, [23]) $A \in(f s: c)$ if and only if (19), (43), and (44) hold and

$$
\lim _{n \rightarrow \infty} \sum_{k}\left|\Delta^{2} a_{n k}\right|=\alpha
$$

(viii) $A \in\left(f s: \ell_{\infty}\right)$ if and only if (43) and (44) hold;

(ix) (Başar and Solak, [24]) $A \in(b s: f s)$ if and only if (44), (45) hold and

$$
\sup _{n \in \mathbb{N}} \sum_{k}|\Delta a(n, k)|<\infty,
$$

$f-\lim a(n, k)=\alpha_{k}$ exists for each fixed $k$;

(x) (Başar, [22]) $A \in(f s: f s)$ if and only if (45), (48) hold and

$\lim _{q \rightarrow \infty} \sum_{k} \frac{1}{q+1} \sum_{i=0}^{q}\left|\Delta^{2}\left[a(n+i, k)-\alpha_{k}\right]\right|=0 \quad$ uniformly in $n$;

(xi) (Başar and Çolak, [21]) $A \in(c s: f s$ ) if and only if (48) holds;

(xii) (Başar, [25]) $A \in(f: c s)$ if and only if

$$
\begin{gathered}
\sup _{n \in \mathbb{N}} \sum_{k}|a(n, k)|<\infty \\
\sum_{n} a_{n k}=\alpha_{k} \quad \text { exists for each fixed } k, \\
\sum_{n} \sum_{k} a_{n k}=\alpha \\
\lim _{m \rightarrow \infty} \sum_{k}\left|\Delta\left[a(n, k)-\alpha_{k}\right]\right|=0 .
\end{gathered}
$$

Now we give our main results which are related to matrix mappings on/into the spaces of almost convergent series $a_{f s}^{r}$ and sequences $a_{f}^{r}$.

Corollary 20. Let $A=\left(a_{n k}\right)$ be an infinite matrix. Then, the following statements hold.

(i) $A \in\left(a_{f s}^{r}: f\right)$ if and only if $\left\{a_{n k}\right\}_{k \in \mathbb{N}} \in\left(a_{f}^{r}\right)^{\beta}$ for all $n \in$ $\mathbb{N}$ and (40), (44) hold with $\widehat{a}_{n k}$ instead of $a_{n k}$, (46) holds with $\widehat{a}(n, k, m)$ instead of $a(n, k, m)$, and (45) holds with $\widehat{a}(n, k)$ instead of $a(n, k)$.

(ii) $A \in\left(a_{f s}^{r}: c\right)$ if and only if $\left\{a_{n k}\right\}_{k \in \mathbb{N}} \in\left(a_{f}^{r}\right)^{\beta}$ for all $n \in \mathbb{N}$ and (19), (43), (44), and (47) hold with $\widehat{a}_{n k}$ instead of $a_{n k}$.

(iii) $A \in\left(a_{f s}^{r}: \ell_{\infty}\right)$ if and only if $\left\{a_{n k}\right\}_{k \in \mathbb{N}} \in\left(a_{f}^{r}\right)^{\beta}$ for all $n \in$ $\mathbb{N}$ and (43) and (44) hold with $\widehat{a}_{n k}$ instead of $a_{n k}$.

(iv) $A \in\left(a_{f s}^{r}: f s\right)$ if and only if $\left\{a_{n k}\right\}_{k \in \mathbb{N}} \in\left(a_{f s}^{r}\right)^{\beta}$ for all $n \in$ $\mathbb{N}$ and (45), (48), and (49) hold with $\widehat{a}(n, k)$ instead of $a(n, k)$. (v) $A \in\left(c s: a_{f_{s}}^{r}\right)$ if and only if (48) holds with $\widetilde{a}(n, k)$ instead of $a(n, k)$.

(vi) $A \in\left(b s: a_{f s}^{r}\right)$ if and only if (44) holds with $\tilde{a}_{n k}$ instead of $a_{n k}$ and (45), (48) hold with $\tilde{a}(n, k)$ instead of $a(n, k)$.

(vii) $A \in\left(f_{s}: a_{f_{s}}^{r}\right)$ if and only if (45), (48), and (49) hold with $\widetilde{a}(n, k)$ instead of $a(n, k)$.

Corollary 21. Let $A=\left(a_{n k}\right)$ be an infinite matrix. Then, the following statements hold.

(i) $A \in\left(c(p): a_{f_{s}}^{r}\right)$ if and only if (37) and (38) hold with $\widetilde{a}(n, k, m)$ instead of $a(n, k, m)$.

(ii) $A \in\left(c_{0}(p): a_{f_{s}}^{r}\right)$ if and only if (37) holds with $\tilde{a}(n$, $k, m)$ instead of $a(n, k, m)$.

(iii) $A \in\left(\ell_{\infty}(p): a_{f_{s}}^{r}\right)$ if and only if (37) and (39) hold with $\widetilde{a}(n, k, m)$ instead of $a(n, k, m)$.

Corollary 22. Let $A=\left(a_{n k}\right)$ be an infinite matrix. Then, the following statements hold.

(i) $A \in\left(a_{f}^{r}: \ell_{\infty}\right)$ if and only if $\left\{a_{n k}\right\}_{k \in \mathbb{N}} \in\left(a_{f}^{r}\right)^{\beta}$ for all $n \in \mathbb{N}$ and (18) holds with $\widehat{a}_{n k}$ instead of $a_{n k}$.

(ii) $A \in\left(a_{f}^{r}: c\right)$ if and only if $\left\{a_{n k}\right\}_{k \in \mathbb{N}} \in\left(a_{f}^{r}\right)^{\beta}$ for all $n \in \mathbb{N}$ and (18), (19), (20), and (21) hold with $\widehat{a}_{n k}$ instead of $a_{n k}$.

(iii) $A \in\left(a_{f}^{r}: c s\right)$ if and only if $\left\{a_{n k}\right\}_{k \in \mathbb{N}} \in\left(a_{f}^{r}\right)^{\beta}$ for all $n \in$ $\mathbb{N}$ and (50),(53) hold with $\widehat{a}(n, k)$ instead of $a(n, k)$ and (51),(52) hold with $\widehat{a}_{n k}$ instead of $a_{n k}$.

Corollary 23. Let $A=\left(a_{n k}\right)$ be an infinite matrix. Then, the following statements hold.

(i) $A \in\left(\ell_{\infty}: a_{f}^{r}\right)$ if and only if (18), (40) hold with $\widetilde{a}_{n k}$ instead of $a_{n k}$ and (41) holds with $\widetilde{a}(n, k, m)$ instead of $a(n, k, m)$.

(ii) $A \in\left(f: a_{f}^{r}\right)$ if and only if (18), (40), and (46) hold with $\widetilde{a}(n, k, m)$ instead of $a(n, k, m)$ and (42) holds with $\widetilde{a}_{n k}$ instead of $a_{n k}$.

(iii) $A \in\left(c: a_{f}^{r}\right)$ if and only if (18), (40), and (42) hold with $\tilde{a}_{n k}$ instead of $a_{n k}$.

(iv) $A \in\left(b s: a_{f}^{r}\right)$ if and only if (40), (43), and (44) hold with $\tilde{a}_{n k}$ instead of $a_{n k}$ and (45) holds with $\tilde{a}(n, k)$ instead of $a(n, k)$.

(v) $A \in\left(f s: a_{f}^{r}\right)$ if and only if (40), (44) hold with $\tilde{a}_{n k}$ instead of $a_{n k}$, (46) holds with $\tilde{a}(n, k, m)$ instead of $a(n$, $k, m)$, and (45) holds with $\widetilde{a}(n, k)$ instead of $a(n, k)$.

(vi) $A \in\left(c s: a_{f}^{r}\right)$ if and only if (40) and (43) hold with $\tilde{a}_{n k}$ instead of $a_{n k}$.

Remark 24. Characterization of the classes $\left(a_{f}^{r}: f_{\infty}\right),\left(f_{\infty}\right.$ : $\left.a_{f}^{r}\right),\left(a_{f s}^{r}: f_{\infty}\right)$, and $\left(f_{\infty}: a_{f_{s}}^{r}\right)$ is redundant since the spaces of almost bounded sequences $f_{\infty}$ and $\ell_{\infty}$ are equal. 


\section{Acknowledgment}

The authors thank the referees for their careful reading of the original paper and for the valuable comments.

\section{References}

[1] J. Boos, Classical and Modern Methods in Summability, Oxford University Press, New York, NY, USA, 2000.

[2] M. Şengönül and F. Başar, "Some new Cesàro sequence spaces of non-absolute type which include the spaces $c_{0}$ and $c$," Soochow Journal of Mathematics, vol. 31, no. 1, pp. 107-119, 2005.

[3] C. Aydın and F. Başar, "Some new sequence spaces which include the spaces $l_{\infty}$ and $l_{P}$," Demonstratio Mathematica, vol. 38, no. 3, pp. 641-656, 2005.

[4] E. Malkowsky and E. Savaş, "Matrix transformations between sequence spaces of generalized weighted means," Applied Mathematics and Computation, vol. 147, no. 2, pp. 333-345, 2004.

[5] M. Mursaleen and A. K. Noman, "On the spaces of $\lambda$-convergent and bounded sequences," Thai Journal of Mathematics, vol. 8, no. 2, pp. 311-329, 2010.

[6] B. Altay, F. Başar, and M. Mursaleen, "On the Euler sequence spaces which include the spaces $l_{p}$ and $l_{\infty} I$," Information Sciences, vol. 176, no. 10, pp. 1450-1462, 2006.

[7] F. Başar and M. Kirişçi, "Almost convergence and generalized difference matrix," Computers \& Mathematics with Applications, vol. 61, no. 3, pp. 602-611, 2011.

[8] A. Sönmez, "Almost convergence and triple band matrix," Mathematical and Computer Modelling, vol. 57, no. 9-10, pp. 23932402, 2013.

[9] K. Kayaduman and M. S. Şengönül, "The space of Cesàro almost convergent sequence and core theorems," Acta Mathematica Scientia, vol. 6, pp. 2265-2278, 2012.

[10] M. Şengönül and K. Kayaduman, "On the Riesz almost convergent sequences space," Abstract and Applied Analysis, vol. 2012, Article ID 691694, 18 pages, 2012.

[11] G. G. Lorentz, "A contribution to the theory of divergent sequences," Acta Mathematica, vol. 80, pp. 167-190, 1948.

[12] I. J. Maddox, "Paranormed sequence spaces generated by infinite matrices," Proceedings of the Cambridge Philosophical Society, vol. 64, pp. 335-340, 1968.

[13] A. Karaisa and F. Özger, "Almost difference sequence space derived by using a generalized weighted mean," Acta Mathematica Scientia. In review.

[14] C. Aydın and F. Başar, "On the new sequence spaces which include the spaces $c_{0}$ and $c$," Hokkaido Mathematical Journal, vol. 33, no. 2, pp. 383-398, 2004.

[15] F. Başar, "A note on the triangle limitation methods," Firat Üniversitesi Mühendislik Bilimleri Dergisi, vol. 5, no. 1, pp. 113-117, 1993.

[16] A. M. Al-Jarrah and E. Malkowsky, "BK spaces, bases and linear operators," in Proceedings of the 3rd International Conference on Functional Analysis and Approximation Theory, vol. 1, no. 52, pp. 177-191, 1998.

[17] A. Wilansky, Summability through Functional Analysis, vol. 85, North-Holland, Amsterdam, The Netherlands, 1984.

[18] J. A. Siddıq1, "Infinite matrices summing every almost periodic sequence," Pacific Journal of Mathematics, vol. 39, pp. 235-251, 1971.

[19] J. P. Duran, "Infinite matrices and almost-convergence," Mathematische Zeitschrift, vol. 128, pp. 75-83, 1972.
[20] J. P. King, "Almost summable sequences," Proceedings of the American Mathematical Society, vol. 17, pp. 1219-1225, 1966.

[21] F. Başar and R. Çolak, "Almost-conservative matrix transformations," Turkish Journal of Mathematics, vol. 13, no. 3, pp. 91-100, 1989.

[22] F. Başar, “ $f$-conservative matrix sequences," Tamkang Journal of Mathematics, vol. 22, no. 2, pp. 205-212, 1991.

[23] E. Öztürk, "On strongly regular dual summability methods," Communications de la Faculte des Sciences de l'Universite d'Ankara, vol. 32, no. 1, pp. 1-5, 1983.

[24] F. Başar and I. Solak, "Almost-coercive matrix transformations," Rendiconti di Matematica e delle sue Applicazioni, vol. 11, no. 2, pp. 249-256, 1991.

[25] F. Başar, "Strongly-conservative sequence-to-series matrix transformations," Erciyes Üniversitesi Fen Bilimleri Dergisi, vol. 5, no. 12 , pp. 888-893, 1989. 


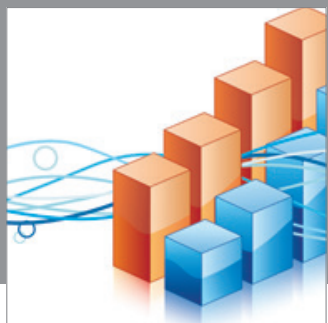

Advances in

Operations Research

mansans

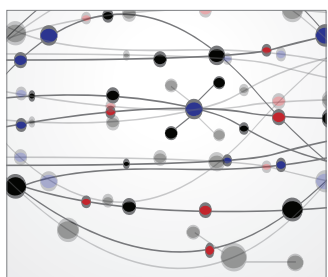

The Scientific World Journal
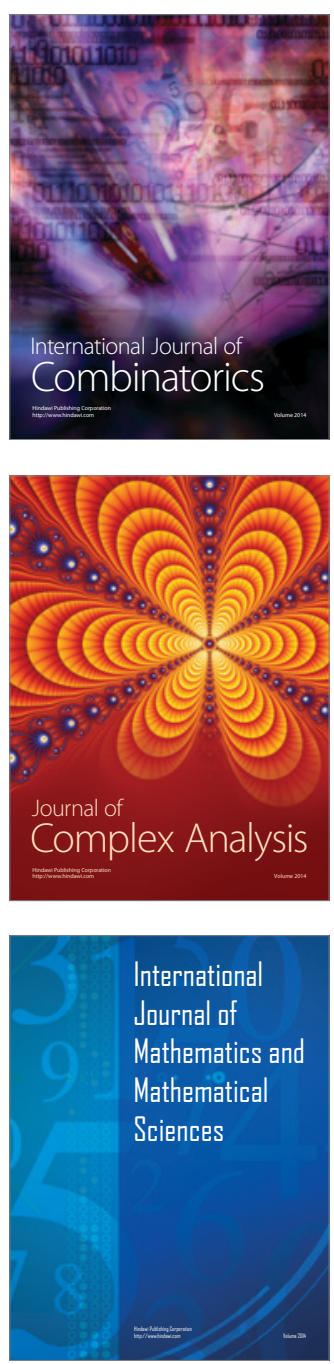
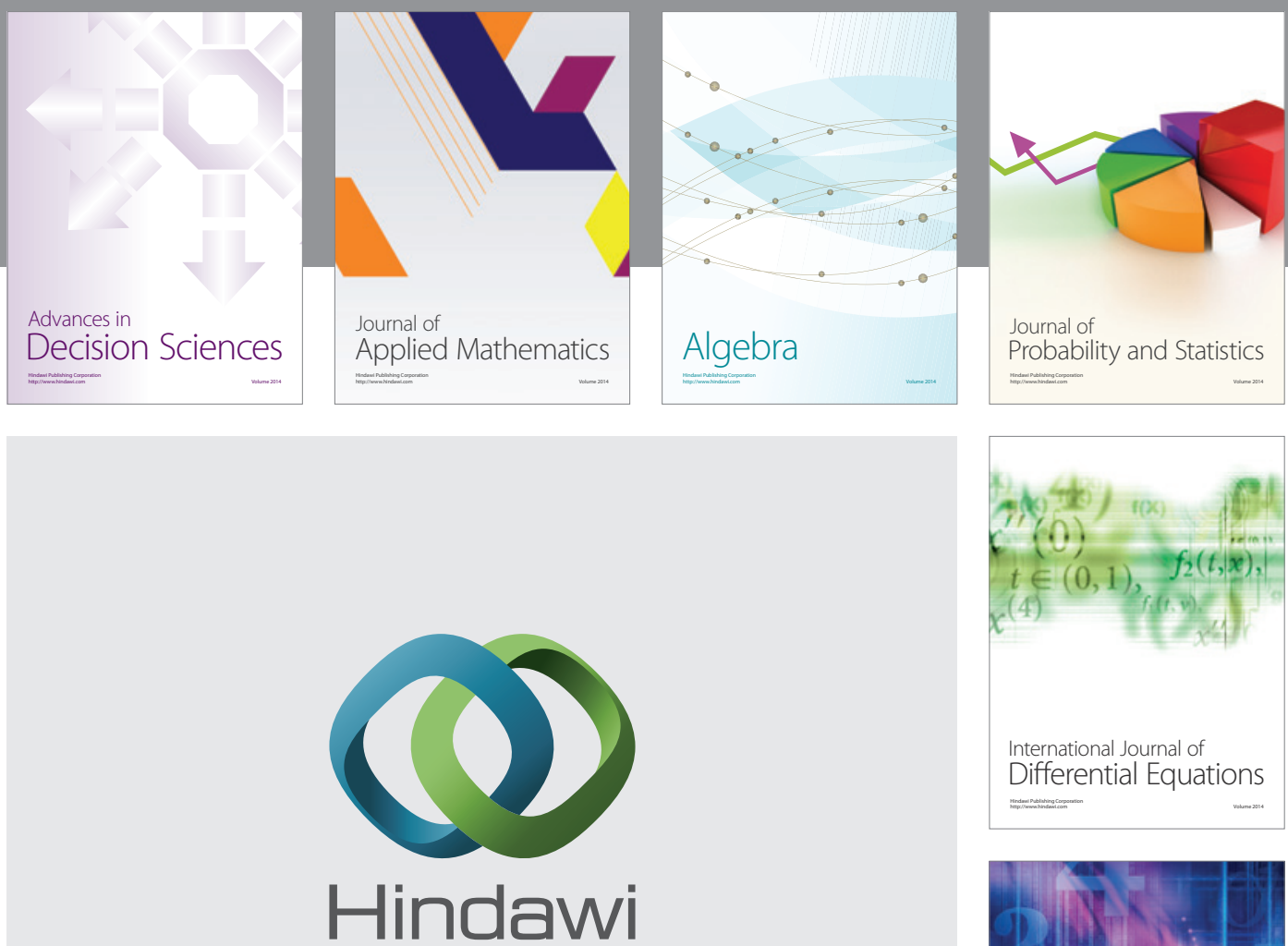

Submit your manuscripts at http://www.hindawi.com
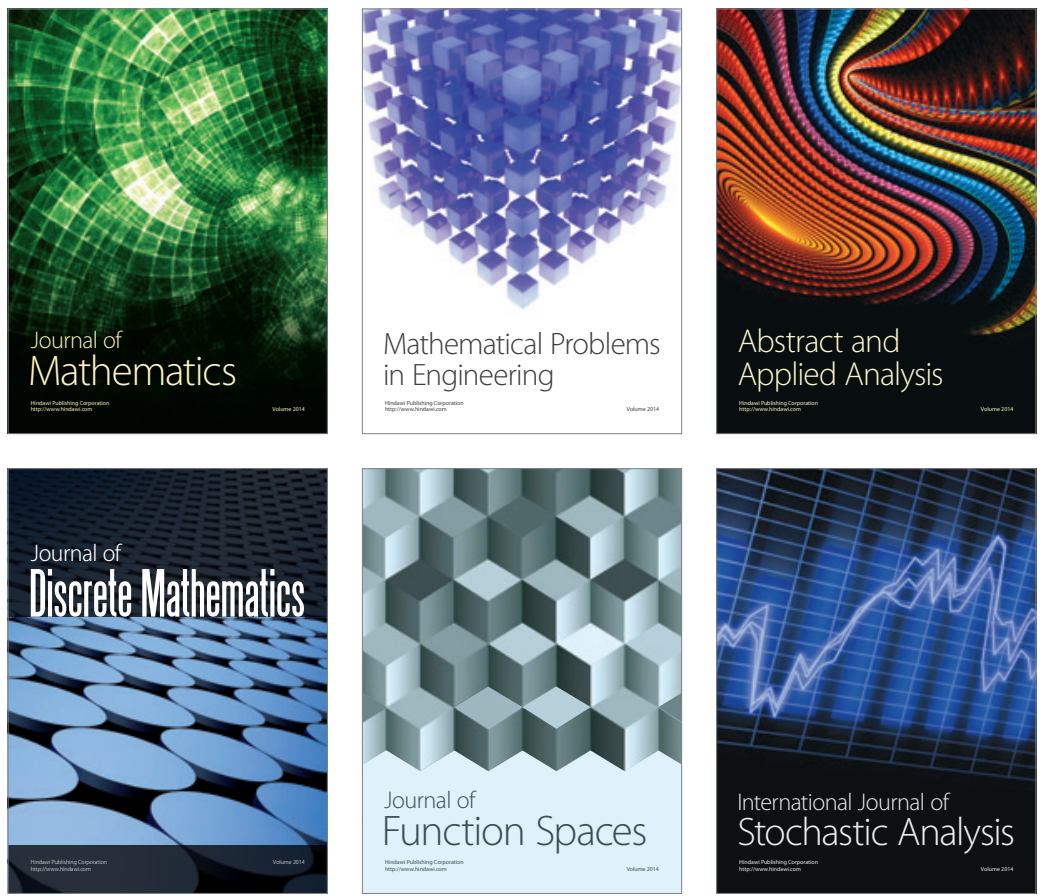

Journal of

Function Spaces

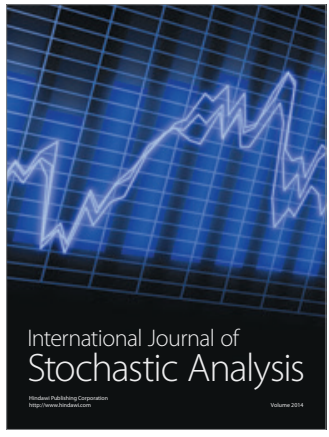

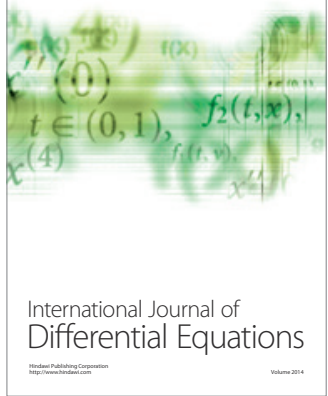
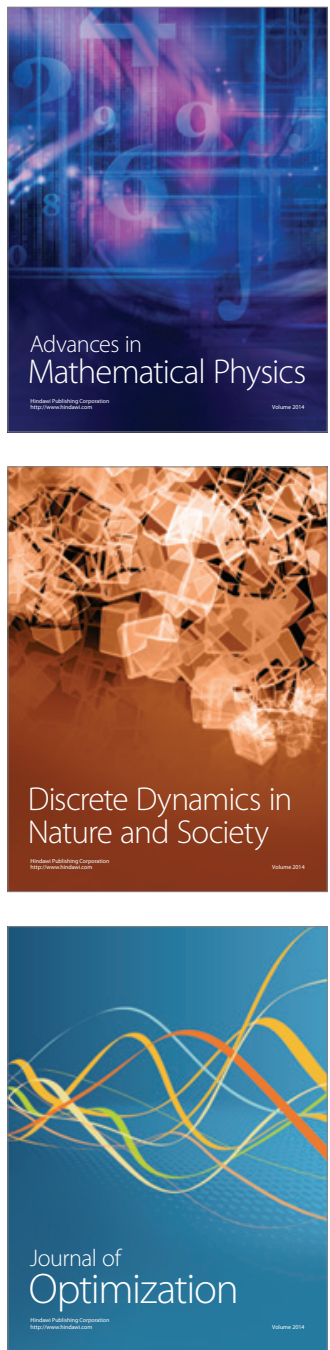